\title{
Nouveaux phosphorothioates dérivés de la naphthylméthylimidazoline et de la naphthyléthylimidazoline : application en radioprotection chimique
}

\author{
B. CÉLARIÈS ${ }^{1}$, C. AMOURETTE ${ }^{2}$, C. LION $^{3}$, G. RIMA ${ }^{1}$
}

(Manuscrit reçu le 16 décembre 2003, accepté le 5 juillet 2004)

RÉSUMÉ Dans ce travail, nous avons synthétisé des phosphorothioates dérivés de la 2-(1-naphthylméthyl)-2-imidazoline et de la 2-[1-(1-naphthyl)éthyl]-2-imidazoline afin d'évaluer, in vivo chez la souris, leur degré de protection contre les effets des rayonnements $\gamma$. Tous ces composés ont montré une activité radioprotectrice remarquable (facteur de réduction de dose compris entre 1,5 et 1,9 ) ce qui les classent parmi les plus actifs actuellement connus dans le domaine de la radioprotection chimique. Ces composés peuvent également présenter un intérêt dans le domaine des traitements de chimio- et de radio-thérapie.

ABSTRACT New phosphorothioates derived from naphthylmethylimidazoline and naphthylethylimidazoline: application in chemical radioprotection.

In this work, we report the radiopharmacological study (radioprotective activity, toxicity) of new phosphorothioates derived from 2-(1-naphthylmethyl)-2imidazoline and 2-[1-(1-naphthyl)ethyl]-2-imidazoline. The radioprotective efficacy against $\gamma$-rays of these organophosphorus compounds was evaluated in vivo. All these derivatives have shown a dose reduction factor between 1.5 and 1.9 which traduce their excellent radioprotective activity. These compounds should present an interest in the chimio- and radiotherapy treatments.

Key words: Radiation therapy / Radioprotectors / Phosphorus compounds

\section{Introduction}

Depuis de nombreuses années la recherche de molécules actives en radioprotection chimique s'était orientée principalement sur une classe chimique : les aminothiols. La plupart de ces dérivés a montré une bonne activité radio-protectrice (cystéamine, ...). Toutefois, leur toxicité n'est pas négligeable. Une nouvelle classe de composés, les phosphorothioates, a présenté un vif intérêt. En effet, ces dérivés

\footnotetext{
1 Laboratoire d'hétérochimie fondamentale et appliquée, université Paul Sabatier, 118 route de Narbonne, 31062 Toulouse Cedex 4, France.

2 Centre de recherches du Service de santé des Armées, 24 avenue des Maquis du Grésivaudan, 38702 La Tronche Cedex, France.

3 ITODYS, Université Paris-VII, 1 rue Guy de la Brosse, 75005 Paris, France.
} 
d'aminothiols ont permis, par la présence d'un groupement $\mathrm{PO}_{3} \mathrm{H}_{2}$ très hydrophile, une meilleure circulation in vivo, en comparaison avec les aminothiols parents, et montrent souvent une activité radio-protectrice nettement supérieure. En effet, les phosphorothioates présentent une activité propre et la plupart de leurs métabolites ont un effet radioprotecteur. Ces composés permettent donc une libération lente et prolongée de substances protectrices.

Le WR-2721 $\left(\mathrm{H}_{2} \mathrm{NCH}_{2} \mathrm{CH}_{2} \mathrm{CH}_{2} \mathrm{NHCH}_{2} \mathrm{CH}_{2} \mathrm{SPO}_{3} \mathrm{X}_{2}\right.$ avec $\left.\mathrm{X}=\mathrm{Li}, \mathrm{H}\right)$ est l'un des meilleurs radioprotecteurs actuellement connus. Il a fait l'objet de nombreuses études en raison de son effet radioprotecteur très élevé (Davidson et al., 1980 ; Piper et al., 1969a, 1969b ; Porta et al., 2000 ; Washburn et al., 1974, 1976 ; Yuhas, 1970, 1972, 1973). Il est actuellement utilisé pour un usage clinique (Amifostine, Ethyol $^{\circledR}$ ) malgré ses nombreux effets secondaires (vomissements dans $25 \%$ des cas, hypotension, etc.). Ce composé protège préférentiellement les cellules saines en raison de sa faible pénétration dans les cellules tumorales.

De plus, l'association du WR-2721 avec la naphthylméthylimidazoline, produit une synergie d'action à des doses injectées auxquelles aucune toxicité n'est observée pour les deux constituants (Laval et al., 1993). Afin de conforter cette idée, nous avons donc synthétisé des phosphorothioates dérivés de la naphthylméthylimidazoline et de la naphthyléthylimidazoline $N$-substituées. Ainsi nous pourrons démontrer qu'il y a une synergie d'action lorsque le couplage se fait de façon chimique. Nous comparerons également les phosphorothioates synthétisés à leurs métabolites aminothiols.

La structure de ces dérivés phosphorés est la suivante :<smiles>[R]CCSP(=O)(O)O</smiles>

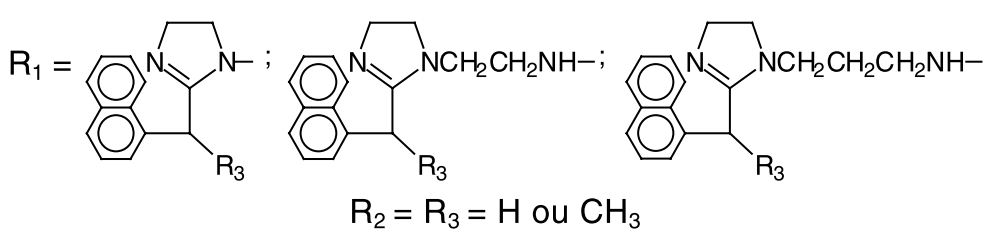

L'addition d'un motif phosphorothioate aux structures organiques permet d'obtenir, pour ces molécules, des propriétés similaires à celles observées lors de l'incorporation d'un atome de silicium ou de germanium dans le cas des composés organométalliques de type métallathiazolidines ou des métalladithioacétals 
développés par notre laboratoire (Célariès et al., 2001a, 2001b, 2003a, 2003b ; Fatôme et al., 1984, 1988 ; Rima et al., 1991, 1993, 1994, 1996, 1997, 1998, 1999a, 1999b ; Satgé et al., 1982, 1989), à savoir :

- la libération in vivo, retardée ou prolongée, d'une ou plusieurs substances organiques possédant des propriétés radio-protectrices (aminothiols) ;

- une modification fondamentale des propriétés chimiques ou biologiques de ces dérivés de base.

L'introduction d'un radical phosphoré hydrophile augmente l'hydrosolubilité et l'activité de ces molécules en favorisant leur transport in vivo. De plus, la présence de groupements naphtaléniques fortement lipophiles favorisent le franchissement des membranes cellulaires.

\section{Technique expérimentale}

Les composés organiques et organophosphorés reportés dans cet article ont été parfaitement caractérisés par les techniques spectroscopiques usuelles et l'analyse centésimale élémentaire. Ils ont été testés purs.

\section{1. Étude radiopharmacologique}

Des souris mâles, Swiss CD1, de poids moyen compris entre 25 et $30 \mathrm{~g}$ à la livraison (élevage Janvier, France) ont été utilisées. Dès leur arrivée, les animaux ont été répartis au hasard par lots de 10 dans des boîtes type III, en makrolon de $33 \times 27 \times 15 \mathrm{~cm}^{3}$. Avant l'expérimentation, les animaux séjournent au moins 7 jours dans l'animalerie maintenue à une température de $21 \pm 1^{\circ} \mathrm{C}$ et à une hygrométrie de $55 \%$; ils sont soumis à un cycle jour-nuit de 12 heures $(8-20 \mathrm{~h})$. Tout au long de l'expérimentation, ils disposent, excepté le temps de l'irradiation, d'eau et de nourriture à volonté.

L'irradiation gamma a été délivrée par une source de ${ }^{60} \mathrm{Co}$. Le débit de dose, à un mètre de la source, était compris entre 50,73 et 52,67 cGy. $\mathrm{min}^{-1}$ en fonction de la date d'expérimentation (décroissance radioactive). Toutes les irradiations se sont déroulées le matin entre 9 et 12 heures. Les souris ont été irradiées par lots de 20 dans une boîte de plexiglas subdivisée en logettes individuelles, plongée dans un champ homogène de $28,5 \times 28,5 \mathrm{~cm}^{2}$ de surface. Le temps de séjour dans les logettes a été limité au temps d'exposition plus 10 minutes. Les animaux étaient positionnés à 1 mètre de la source. La dose à mi-corps dans l'air a été contrôlée par une chambre d'ionisation Baldwin-Ionex. Pour chaque produit testé, 10 animaux ont été exposés à une dose qui conduit à la mort de tous les animaux témoins au cours des trente jours qui suivent l'irradiation ( $\mathrm{DL}_{100 / 30}$ jours $)$ et 10 animaux ont 
été exposés à une dose de 2 Gy supérieure. $\mathrm{La} \mathrm{DL}_{100 / 30}$ jours a préalablement été déterminée par la méthode des «probits » (Bonet-Maury et al., 1950 ; Finney, 1971), en soumettant plusieurs lots d'animaux à des doses d'irradiation croissantes et en traçant la courbe de létalité correspondante. Cette courbe est retracée tous les six mois environ.

Lors de chaque journée d'irradiation, des contrôles sont effectués : 5 souris recevant le miglyol et 5 souris non traitées sont irradiées à la $\mathrm{DL}_{100 / 30}$ jours ; pour chaque produit testé la survie est suivie pendant 30 jours sur 5 souris qui n'ont pas été irradiées afin de vérifier qu'il n'y a pas de toxicité à long terme.

Les composés testés ont été dissous dans un solvant inerte et non toxique, le miglyol (mélange d'ester d'acides gras), avant d'être injectés par voie intrapéritonéale (ip) à l'animal, soit $15 \mathrm{~min}$, soit $90 \mathrm{~min}$ avant l'irradiation (selon la structure de la molécule). La dose de produit injecté était égale à la moitié de la $\mathrm{DL}_{50}$ toxicologie déterminée au préalable ; le volume injecté était de $500 \mu \mathrm{L}$. La mortalité a été suivie deux fois par jour, pendant les 30 jours suivant l'exposition.

Pour chacun des produits testés et pour chaque dose d'exposition, la courbe de survie en fonction du temps a été tracée afin de déterminer le taux de survie à 30 jours, le temps moyen de survie (TMS ou moyenne arithmétique des durées de vie de chaque animal entre le début et la fin de l'irradiation) et le temps de survie $50 \%$ (TS $50 \mathrm{ou}$, pour un lot de $\mathrm{N}$ animaux, le temps écoulé entre le début de l'expérience et la mort du «(N-ième/2 )+1 » animal).

Pour les trois composés les plus actifs (afin de limiter le nombre d'animaux utilisés), des irradiations supplémentaires (20 souris par dose et par produit) à 7,5, $8,1,10,1,11,1,12,1,14,1$ et 16,1 Gy ont été réalisées. Pour chacun de ces trois produits et pour des animaux témoins irradiés et non traités, en traçant la droite mortalité $=f[\log ($ dose $)]$, nous avons déterminé la $\mathrm{DL}_{50 / 30}$ jours afin de calculer le facteur de réduction de dose.

\section{Résultats et discussion}

Les phosphorothioates ont été obtenus par action de $\mathrm{Na}_{3} \mathrm{SPO}_{3}$ sur les dérivés bromés de la naphthylméthylimidazoline ou de la naphthyléthylimidazoline $N$-substituée, dans un mélange eau/DMF à $40^{\circ} \mathrm{C}$ (Piper et al., 1969a, 1969b).

Dans ces différents composés, nous allons pouvoir estimer l'influence du phosphore, par comparaison entre les phosphorothioates et leurs équivalents aminothiols. Également, nous pouvons comparer les dérivés de la naphthylméthylimidazoline et leurs homologues de la naphthyléthylimidazoline. 
TABLEAU I

Données toxicologiques et taux de survie des composés 1-6. Toxicological data and survival rate of compounds 1-6.

Phosphorothioates dérivés de la cystéamine $N$-substituée $\mathrm{R}_{1} \mathrm{CH}_{2} \mathrm{CH}_{2} \mathrm{~S}-\stackrel{\mathrm{P}}{\|}-\mathrm{OH}$

\begin{tabular}{|c|c|c|c|c|c|}
\hline $\mathbf{N}^{\circ}$ & $\mathbf{R}_{1}$ & $\begin{array}{c}\mathrm{DL}_{50}: \mathrm{mg} \mathrm{kg}^{-1} \\
(\mathrm{mmol})\end{array}$ & $\begin{array}{c}\text { Dose injectée } \\
\mathrm{mg} \mathrm{kg}^{-1}\end{array}$ & $\begin{array}{l}\text { Irradiation : } \\
\text { Gy }(t, \text { min })^{a}\end{array}$ & $\begin{array}{c}\text { Taux de } \\
\text { survie (\%) }\end{array}$ \\
\hline 1 & & $168(0,48)$ & 84 & $\begin{array}{r}8,1(15) \\
10,1(15)\end{array}$ & $\begin{array}{r}100 \\
70\end{array}$ \\
\hline 2 & & $184(0,50)$ & 92 & $\begin{array}{r}8,1(15) \\
10,1(15)\end{array}$ & $\begin{array}{r}100 \\
80\end{array}$ \\
\hline 3 & & $280(0,71)$ & 140 & $\begin{array}{r}8,1(15) \\
10,1(15)\end{array}$ & $\begin{array}{c}10 \\
0\end{array}$ \\
\hline 4 & & $400(0,98)$ & 200 & $\begin{array}{r}8,1(15) \\
10,1(15)\end{array}$ & $\begin{array}{l}90 \\
60\end{array}$ \\
\hline 5 & & $150(0,37)$ & 75 & $\begin{array}{r}8,1(15) \\
10,1(15)\end{array}$ & $\begin{array}{c}100 \\
50\end{array}$ \\
\hline 6 & & $260(0,62)$ & 130 & $\begin{array}{r}8,1(15) \\
10,1(15)\end{array}$ & $\begin{array}{r}100 \\
70\end{array}$ \\
\hline
\end{tabular}

${ }^{\mathrm{a}} \mathrm{t}=$ temps écoulé entre l'injection du radioprotecteur par voie intrapéritonéale et l'irradiation.

\subsection{Influence du phosphore}

Le phosphore n'a pas une grande influence sur la toxicité. En effet, les phosphorothioates ( $c f$. Tabs. I et II) et leurs équivalents aminothiols (Tabs. III et IV) présentent des $\mathrm{DL}_{50}$ (ramenées à la même quantité injectée : $\mathrm{mmol} \cdot \mathrm{kg}^{-1}$ ) du 
TABLEAU II

Données toxicologiques et taux de survie des composés 7-10. Toxicological data and survival rate of compounds 7-10.

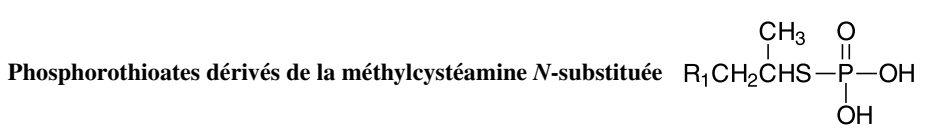

\begin{tabular}{|c|c|c|c|c|c|}
\hline $\mathbf{N}^{\circ}$ & $\mathbf{R}_{1}$ & $\begin{array}{c}\mathrm{DL}_{50}: \mathrm{mg} \cdot \mathrm{kg}^{-1} \\
(\mathrm{mmol})\end{array}$ & $\begin{array}{l}\text { Dose injectée } \\
\mathrm{mg} \cdot \mathrm{kg}^{-1}\end{array}$ & $\begin{array}{l}\text { Irradiation : } \\
\text { Gy }(t, \text { min })^{a}\end{array}$ & $\begin{array}{c}\text { Taux de } \\
\text { survie (\%) }\end{array}$ \\
\hline 7 & & $184(0,50)$ & 92 & $\begin{array}{r}8,1(15) \\
10,1(15)\end{array}$ & $\begin{array}{c}100 \\
70\end{array}$ \\
\hline 8 & & $260(0,69)$ & 130 & $\begin{array}{r}8,1(15) \\
10,1(15)\end{array}$ & $\begin{array}{c}40 \\
0\end{array}$ \\
\hline 9 & & $150(0,37)$ & 75 & $\begin{array}{r}8,1(15) \\
10,1(15)\end{array}$ & $\begin{array}{l}80 \\
70\end{array}$ \\
\hline 10 & & $360(0,85)$ & 180 & $\begin{array}{r}8,1(15) \\
10,1(15)\end{array}$ & $\begin{array}{c}100 \\
70\end{array}$ \\
\hline
\end{tabular}

${ }^{\mathrm{a}} \mathrm{t}$ = temps écoulé entre l'injection du radioprotecteur par voie intrapéritonéale et l'irradiation.

même ordre de grandeur. Par contre, les prodrogues phosphorées, en comparaison avec les aminothiols, possèdent une excellente activité radio-protectrice. Ces molécules présentent, probablement, un bon compromis entre leur caractère lipophile et hydrophile.

\subsection{Comparaison des substituants (cf. Tabs. V et VI)}

Tout ce qui pourra être dit concernant les substituants, se rapporte à leurs effets dans les structures organométalliques et, en aucun cas, lorsqu'ils sont isolés.

Les dérivés de la naphthyléthylimidazoline sont plus actifs et moins toxiques que leurs homologues de la naphthylméthylimidazoline 1. Le composé 4 
TABLEAU III

Données toxicologiques et taux de survie des aminothiols 1'-4' (métabolites des composés 1-4). Toxicological data and survival rate of aminothiols 1'-4' (metabolites of compounds 1-4).

Aminothiols dérivés de la cystéamine $N$-substituée $\mathrm{R}_{1} \mathrm{CH}_{2} \mathrm{CH}_{2} \mathrm{SH}$

\begin{tabular}{|c|c|c|c|c|c|}
\hline $\mathbf{N}^{\circ}$ & $\mathbf{R}_{1}$ & $\underset{(\mathrm{mmol})}{\mathrm{DL}_{50}: \mathrm{mg}^{-\mathrm{kg}^{-1}}}$ & $\begin{array}{c}\text { Dose injectée } \\
\mathrm{mg} \mathrm{kg}^{-1}\end{array}$ & $\begin{array}{l}\text { Irradiation : } \\
\text { Gy }(t, \text { min })^{\mathrm{a}}\end{array}$ & $\begin{array}{c}\text { Taux de } \\
\text { survie }(\%)\end{array}$ \\
\hline 1 ' & & $\begin{array}{l}35(0,13) \\
\text { FRD }=1,1\end{array}$ & 17,5 & $\begin{array}{r}9,75(15) \\
11,75(15)\end{array}$ & $\begin{array}{c}40 \\
0\end{array}$ \\
\hline 2 ' & & $>150(0,53)$ & 75 & $\begin{array}{r}8,4(15) \\
10,4(15)\end{array}$ & $\begin{array}{c}30 \\
0\end{array}$ \\
\hline 3 , & & $\begin{array}{l}125(0,40) \\
\text { FRD }=1,1\end{array}$ & 62,5 & $\begin{array}{r}9,75(15) \\
11,75(15)\end{array}$ & $\begin{array}{c}30 \\
0\end{array}$ \\
\hline 4 ' & & $>300(0,92)$ & 150 & $\begin{array}{r}8,1(15) \\
10,1(15)\end{array}$ & $\begin{array}{l}0 \\
0\end{array}$ \\
\hline
\end{tabular}

${ }^{\mathrm{a}} \mathrm{t}$ = temps écoulé entre l'injection du radioprotecteur par voie intrapéritonéale et l'irradiation.

présente la plus faible toxicité de cette série. En effet, le prolongement de la chaîne semble bénéfique puisqu'il est presque deux fois moins toxique que le dérivé 2. Le motif WR-2721 (N-C-C-C-N-C-C-S-P) s'avère moins efficace et plus toxique lorsqu'il est couplé à la naphthylméthylimidazoline plutôt qu'à la naphthyléthylimidazoline. Les excellents taux de survie, obtenus avec des doses injectées relativement faibles, rappellent la synergie d'action que nous constatons lorsque les deux composés (WR-2721 et naphthylméthylimidazoline) sont injectés simultanément (Laval et al., 1993). La majorité de ces composés possède une activité encore très forte lorsque nous irradions à $10,1 \mathrm{~Gy}$ $\left(\mathrm{DL}_{100 / 30}\right.$ jours $\left.+2 \mathrm{~Gy}\right)$. Dans cette série, nous avons déterminé le facteur de réduction de dose du composé $\mathbf{6}$ et nous le comparerons, par la suite, à celui des composés $\mathbf{7}$ et $\mathbf{1 0}$. 


\section{TABLEAU IV}

Données toxicologiques et taux de survie des aminothiols 7'-10' (métabolites des composés 7-10). Toxicological data and survival rate of aminothiols $7^{\prime}-10$ ' (metabolites of compounds 7-10).

$\mathrm{CH}_{3}$ Aminothiols dérivés de la méthylcystéamine $N$-substituée $\quad \mathrm{R}_{1} \mathrm{CH}_{2} \mathrm{CHSH}$

\begin{tabular}{|c|c|c|c|c|c|}
\hline $\mathbf{N}^{\circ}$ & $\mathbf{R}_{1}$ & $\underset{(\mathrm{mmol})}{\mathrm{DL}_{50}: \mathrm{mg}^{-\mathrm{kg}^{-1}}}$ & $\begin{array}{l}\text { Dose injectée } \\
\mathrm{mg} \mathrm{kg}^{-1}\end{array}$ & $\begin{array}{l}\text { Irradiation : } \\
\text { Gy }(t, \min )^{\mathrm{a}}\end{array}$ & $\begin{array}{c}\text { Taux de } \\
\text { survie }(\%)\end{array}$ \\
\hline 7 & & $\begin{array}{l}71(0,28) \\
\text { FRD }=1,1\end{array}$ & 35,5 & $\begin{array}{r}9,75(15) \\
11,75(15)\end{array}$ & $\begin{array}{r}35 \\
0\end{array}$ \\
\hline 8 ' & & $>300(1,01)$ & 150 & $\begin{array}{r}8,4(15) \\
10,4(15)\end{array}$ & $\begin{array}{l}0 \\
0\end{array}$ \\
\hline 9' & & $\begin{array}{l}80(0,24) \\
F R D=1,3\end{array}$ & 62,5 & $\begin{array}{r}9,75(15) \\
11,75(15)\end{array}$ & $\begin{array}{l}60 \\
40\end{array}$ \\
\hline 10 ' & & $212(0,62)$ & 106 & $\begin{array}{r}8,1(15) \\
10,1(15)\end{array}$ & $\begin{array}{l}0 \\
0\end{array}$ \\
\hline
\end{tabular}

${ }^{\mathrm{a}} \mathrm{t}=$ temps écoulé entre l'injection du radioprotecteur par voie intrapéritonéale et l'irradiation.

Les phosphorothioates dérivés de la naphthyléthylimidazoline possèdent une toxicité plus faible et une activité plus importante. Les dérivés prolongés $(\mathbf{9 , 1 0})$ sont également moins toxiques que $\mathbf{7}$ et $\mathbf{8}$. Le composé $\mathbf{2}$ a montré la meilleure activité de la série. La substitution d'un atome d'hydrogène, dans $\mathbf{2}$, par un groupement méthyle, pour conduire à $\mathbf{8}$, induit une chute très importante de l'activité et même une perte d'effet radioprotecteur pour une irradiation à une dose de 10,1 Gy. À l'inverse, dans le cas du phosphorothioate $\mathbf{9}$, la présence du méthyle permet une augmentation très nette du pouvoir radioprotecteur, par rapport au dérivé 3 qui était le moins actif de la série.

Globalement les dérivés méthylés en $\alpha$ du soufre, sont moins toxiques que leurs homologues non méthylés. L'influence sur l'activité radio-protectrice est 
TABLEAU V

Comparaison des différents substituants des composés 1-6. Comparison of the several substituents of compounds 1-6.

Comparaison des différents substituants : (C) $\mathrm{CH}_{2} \mathrm{CH}_{2} \mathrm{~S}-\stackrel{\mathrm{II}}{\mathrm{P}}-\mathrm{OH}$

\begin{tabular}{|c|c|c|c|c|}
\hline $\mathbf{N}^{\circ}$ & Substituant L & $\begin{array}{c}\mathrm{DL}_{50}: \mathrm{mg} \mathrm{kg}^{-1} \\
(\mathrm{mmol})\end{array}$ & $\begin{array}{c}\text { irradiation } \\
\left(\text { Gy) }(\mathbf{t}, \mathbf{m i n})^{\mathrm{a}}\right.\end{array}$ & Survie (\%) \\
\hline 1 & & $168(0,48)$ & $\begin{array}{r}8,1(15) \\
10,1(15)\end{array}$ & $\begin{array}{c}100 \\
70\end{array}$ \\
\hline 2 & & $184(0,50)$ & $\begin{array}{r}8,1(15) \\
10,1(15)\end{array}$ & $\begin{array}{c}100 \\
80\end{array}$ \\
\hline 3 & & $280(0,71)$ & $\begin{array}{r}8,1(15) \\
10,1(15)\end{array}$ & $\begin{array}{c}10 \\
0\end{array}$ \\
\hline 4 & & $400(0,98)$ & $\begin{array}{r}8,1(15) \\
10,1(15)\end{array}$ & $\begin{array}{l}90 \\
60\end{array}$ \\
\hline 5 & & $150(0,37)$ & $\begin{array}{r}8,1(15) \\
10,1(15)\end{array}$ & $\begin{array}{c}100 \\
50\end{array}$ \\
\hline 6 & & $260(0,62)$ & $\begin{array}{r}8,1(15) \\
10,1(15)\end{array}$ & $\begin{array}{c}100 \\
70\end{array}$ \\
\hline
\end{tabular}

${ }^{a} \mathrm{t}=$ temps écoulé entre l'injection du radioprotecteur par voie intrapéritonéale et l'irradiation. 


\section{TABLEAU VI}

Comparaison des différents substituants des composés 7-10. Comparison of the several substituents of compounds 7-10.

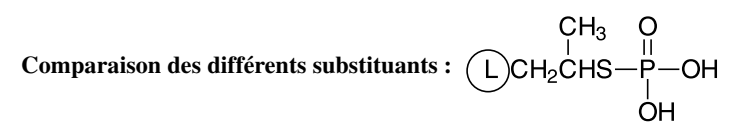

\begin{tabular}{|c|c|c|c|c|}
\hline $\mathbf{N}^{\circ}$ & Substituant L & $\underset{(\mathrm{mmol})}{\mathrm{DL}_{50}: \mathrm{mg}^{-\mathrm{kg}^{-1}}}$ & $\begin{array}{c}\text { irradiation } \\
\left(\text { Gy) }(\mathbf{t}, \mathbf{m i n})^{\mathrm{a}}\right.\end{array}$ & Survie (\%) \\
\hline 7 & & $184(0,50)$ & $\begin{array}{r}8,1(15) \\
10,1(15)\end{array}$ & $\begin{array}{c}100 \\
70\end{array}$ \\
\hline 8 & & $260(0,69)$ & $\begin{array}{r}8,1(15) \\
10,1(15)\end{array}$ & $\begin{array}{c}40 \\
0\end{array}$ \\
\hline 9 & & $150(0,37)$ & $\begin{array}{c}8,1(15) \\
10,1(15)\end{array}$ & $\begin{array}{l}80 \\
70\end{array}$ \\
\hline 10 & & $360(0,85)$ & $\begin{array}{r}8,1(15) \\
10,1(15)\end{array}$ & $\begin{array}{c}100 \\
70\end{array}$ \\
\hline
\end{tabular}

${ }^{a} \mathrm{t}=$ temps écoulé entre l'injection du radioprotecteur par voie intrapéritonéale et l'irradiation.

moins systématique. Nous pouvons classer ces substituants suivant l'effet qu'ils apportent aux structures organophosphorées :

\section{effet radioprotecteur :}

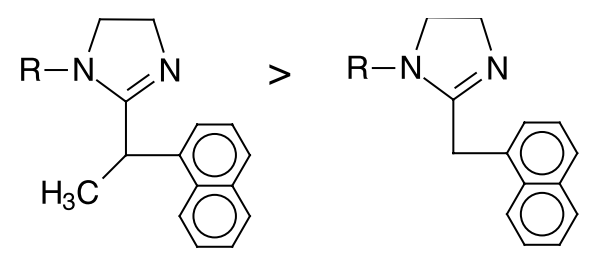


toxicité :

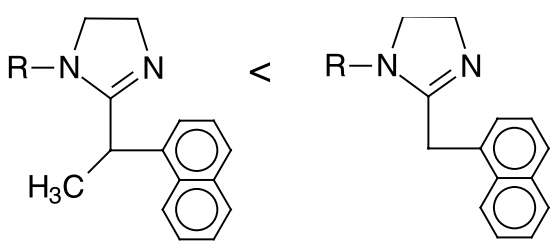

\subsection{Calcul du facteur de réduction de dose}

Nous avons choisi de calculer les facteurs de réduction de dose uniquement pour quelques composés $\left(\mathbf{6}, 7\right.$ et 10) qui présentaient une toxicité faible $\left(\mathrm{DL}_{50} \geq\right.$ $0,50 \mathrm{mmol} \cdot \mathrm{kg}^{-1}$ ) et avaient donné une bonne activité préliminaire (au moins $100 \%$ de survie pour une irradiation de 8,1 Gy et $70 \%$ pour $10,1 \mathrm{~Gy}$ ) afin de limiter le nombre d'animaux utilisé. Il est bon de rappeler que le facteur de réduction de dose mesure quantitativement le pouvoir radioprotecteur d'une substance donnée. Il est défini comme le rapport des doses d'irradiation qui provoquent $50 \%$ de mortalité respectivement chez les animaux protégés et les animaux non protégés :

Facteur de réduction de dose $=\frac{\mathrm{DL}_{50 / 30} \text { jours } \text { irradiation (animaux protégés) }}{\mathrm{DL}_{50 / 30} \text { jours } \text { irradiation (animaux non protégés) }}$

Afin de déterminer la $\mathrm{DL}_{50}$ d'irradiation, il faut tracer la droite de mortalité en fonction du logarithme décimal de la dose (cf. Fig. 1).

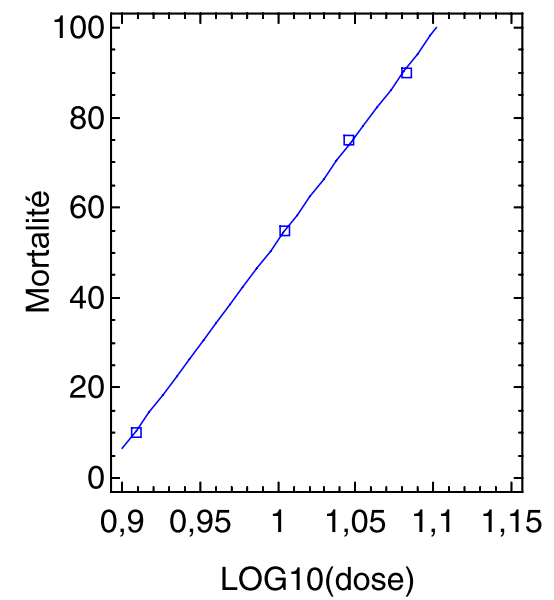

Figure 1 - Tracé mortalité $=f[\log ($ Dose $)]$ pour le composé 10.

Mortality $=f[\log ($ Dose $)]$ for compound 10 . 


\section{TABLEAU VII}

Détermination du facteur de réduction de dose des composés 6,7 et 10. Determination of the dose reduction factor of compounds 6,7 et 10 .

Facteur de réduction de dose des composés 6, 7, $10: \mathrm{R}_{1} \mathrm{CH}_{2} \mathrm{CHS} \stackrel{\mathrm{R}_{2}}{\stackrel{\mathrm{I}}{\mathrm{P}}-\mathrm{OH}}$

\begin{tabular}{ccccccc}
\hline $\mathbf{N}^{\circ}$ composé & $\mathbf{R}_{\mathbf{1}}$ & $\mathbf{R}_{\mathbf{2}}$ & $\begin{array}{c}\text { Coefficient de } \\
\text { corrélation }\end{array}$ & $\begin{array}{c}\mathbf{D L}_{\mathbf{5 0}} \\
\text { irradiation (Gy) }\end{array}$ & FRD \\
\hline Témoins & -- & 0,96996 & $6,22 \pm 0,14$ & --- \\
\hline & & & & & & \\
\end{tabular}

Afin d'obtenir cette droite, pour chaque produit, nous avons soumis plusieurs lots de 20 souris à des doses d'irradiation croissantes : 7,5;8,1;10,1;11,1;12,1; 14,1 et $16,1 \mathrm{~Gy}$. De la même façon, $\mathrm{la}_{\mathrm{DL}}{ }_{50}$ d'irradiation pour les animaux témoins est vérifiée en utilisant des doses de $7 ; 7,5$ et 8,1 Gy. La détermination des $\mathrm{DL}_{50}$ a été réalisée à l'aide d'un logiciel de calculs statistiques (STATGRAPHICS Plus 5.1) permettant de faire des régressions linéaires et log-probit. Les valeurs des $\mathrm{DL}_{50}$ et des facteurs de réduction de dose sont reportées dans le tableau VII.

Ces trois composés possèdent de très bons facteurs de protection. Le plus actif des trois semble être le composé 7, qui est un dérivé de la naphthylméthylimidazoline. Les deux autres composés présentent à peu près le même facteur de réduction de dose.

Ces trois composés présentaient les mêmes taux de survie (100\% pour 8,1 Gy et $70 \%$ pour $10,1 \mathrm{~Gy}$ ) lors des tests préliminaires et pourtant leurs facteurs de réduction de dose sont différents. Ceci traduit la difficulté qu'il y a à comparer des taux de survie et donc à extrapoler la valeur des facteurs de réduction de dose des 
autres phosphorothioates à partir de ceux de ces trois dérivés. Nous pouvons cependant tenter de donner une limite inférieure et supérieure à l'activité des autres dérivés phosphorés. Ainsi :

- $\quad 1,5<$ facteur de réduction de dose $<1,85$ pour les composés $\mathbf{1}, \mathbf{2}, \mathbf{5}, \mathbf{6}, \mathbf{7}$ et $\mathbf{1 0}$ car leurs taux de survie, lors des tests préliminaires, étaient voisins $(100 \%$ à $8,1$ Gy et $\geq 50 \%$ à $10,1 \mathrm{~Gy})$;

- $\quad 1,3<$ facteur de réduction de dose $<1,5$ pour les molécules 4 et $9(80-90 \%$ à 8,1 Gy et 70-60\% à 10,1 Gy);

- les phosphorothioates $\mathbf{3}$ et $\mathbf{8}$ ne présentent qu'une faible activité radioprotectrice.

\section{Conclusion}

La démarche que nous avons entreprise, dans cet article, était d'établir une relation structure-activité radioprotectrice à partir d'un nombre limité de molécules.

Cette analyse met en évidence l'activité radio-protectrice notable qu'apportent les composés phosphorés synthétisés. L'étude de l'influence des différents substituants a fait ressortir que les dérivés de la naphthyléthylimidazoline sont moins toxiques et surtout plus actifs que leurs homologues de la naphthylméthylimidazoline. La présence d'un groupement méthyle en $\alpha$ du soufre n'a pas une influence systématique, sur la toxicité ou l'effet radioprotecteur.

La substitution d'un proton sur le pont $\mathrm{CH}_{2}$ de la naphthylméthylimidazoline, par un groupement alkyle, apparaît ainsi plus favorable dans l'amélioration des propriétés radioprotectrices que celle d'un proton en $\alpha$ du soufre.

Les phosphorothioates présentent une toxicité voisine de celle des aminothiols parents mais, par contre, possèdent une activité radio-protectrice très supérieure. Ceci montre la contribution favorable du phosphore dans l'établissement du pouvoir radioprotecteur. Cette constatation met en évidence la possibilité de potentialisation des substances organiques de base dans l'organisme de la souris, grâce aux substituants phosphorés hydrophiles. De plus nous avons retrouvé la synergie d'action, qui apparaissait lorsque le WR-2721 et la naphthylméthylimidazoline étaient injectés simultanément (Laval et al., 1993), en couplant ces deux composés chimiquement.

Parmi les dix composés synthétisés et dans un souci d'économie d'animaux, nous avons seulement réalisé la détermination du facteur de réduction de dose de trois dérivés. Le composé 7 présente un facteur de réduction de dose de l'ordre de 1,85 et les phosphorothioates $\mathbf{6}$ et $\mathbf{1 0}$ un facteur de réduction de dose d'environ 1,60 et nous pouvons tenter d'extrapoler les résultats des sept autres dérivés 
phosphorés. Sur les dix molécules, six composés sont susceptibles d'avoir un facteur de réduction de dose supérieur à 1,5 et le phosphorothioate 2 pourrait présenter un facteur de réduction de dose supérieur à 1,85 car il offrait la meilleure protection lors des tests préliminaires.

Remerciements. Les auteurs souhaitent remercier la Délégation générale pour l'Armement (DGA/DSP/STTC/DT/SH), ministère de la Défense nationale, France, pour leur soutien financier et pour l'intérêt porté à cette recherche.

\section{RÉFÉRENCES}

Bonet-Maury P., Patti F. (1950) L'irradiation mortelle de la souris par les rayons X et $\gamma$. Essais thérapeutiques, J. Radiol. Electrol. Arch. Electr. Méd. 31, 286-290.

Célariès B., Court L., Lion C., Rima G., Roman V. (2001a) Organometallic compounds of silicon and germanium in chemical radioprotection, Main Group Met. Chem. 25, 58.

Célariès B., Rima G., Court L., Lion C., Roman V., Laval J.D. (2001b) Radioprotective activity and synthesis of siladithioacetals and germadithioacetals derived from $\mathrm{N}$-substituted naphthylethylimidazoline, Metal-Based Drugs 8, 199-210.

Célariès B., Amourette C., Lion C., Rima G. (2003a) Radioprotective activity of metalladithioacetals derived from $N$-substituted naphthylethylimidazoline, Appl. Organomet. Chem. 17, 135-138.

Célariès B., Amourette C., Lion C., Rima G. (2003b) Synthesis and radioprotective study of new siladithioacetals and germadithioacetals, Appl. Organomet. Chem. 17, 561-569.

Davidson D.E., Grenan M.M., Sweeney T.R. (1980) Radiation Sensitizers. Their use in the clinical management of cancer (Luther W. Brady, Ed.) p. 309. Masson pub., USA.

Fatôme M., Sentenac-Roumanou H., Lion C., Satgé J., Fourtinon M., Rima G. (1984) Radioprotective effect of germanium dithioacetals of cysteamine and methylcysteamine, Eur. J. Med. Chem. 19, $119-122$

Fatôme M., Sentenac-Roumanou H., Lion C., Satgé J., Rima G. (1988) Study of germathiazolidines and germylated dithioacetals derived from $N$-substituted cysteamine and methylcysteamine: synthesis and radioprotective activity, Eur. J. Med. Chem. 23, 257-266.

Finney D.J. (1971) Probit. Analysis: a statistical treatment of the sigmoid response curve, 3rd edn. Cambridge University Press.

Laval J.D., Roman V., Laduranty J., Miginiac L., Lion C., Sentenac-Roumanou H., Fatôme M. (1993) Radioprotective effect of low doses of 2-(1-naphthylmethyl)-2-imidazoline alone or associated with phosphorothioates, Eur. J. Med. Chem. 28, 709-713.

Piper J.R., Stringfellow C.R., Elliot R.D., Johnston T.P. (1969a) S-2-(omega-aminoalkylamino)ethyl dihydrogen phosphorothioates and related compounds as potential antiradiation agents, $J$. Med. Chem. 12, 236-243.

Piper J.R., Stringfellow C.R., Johnston T.P. (1969b) Terminally substituted S-2-(omegaaminoalkylamino)ethyl dihydrogen phosphorothioates and related compounds as potential antiradiation agents, J. Med. Chem. 12, 244-253.

Porta C., Maiolo A., Tua A., Grignani G. (2000) Amifostine, a reactive oxygen species scavenger with radiation- and chemo-protective properties, inhibits in vitro platelet activation induced by ADP, collagen or PAF, Haematologica. 85, 820-825.

Rima G., Satgé J., Fatôme M., Laval J.D., Sentenac-Roumanou H., Lion C., Lazraq M. (1991) Syntheses and radioprotective activities of trithiagermatranes, trithiasilatranes, germylated oxide and sulfide with cysteamine and $N$-(2-thioethyl)-1,3-diaminopropane hydrochloride ligands, Eur. J. Med. Chem. 26, 291-297. 
Rima G., Satgé J., Sentenac-Roumanou H., Fatôme M., Lion C., Laval J.D. (1993) New organogermylated compounds $N$-substituted by naphthylmethylimidazoline hydrochloride, and imidazoline: synthesis and radioprotective activity, Eur. J. Med. Chem. 28, 761-767.

Rima G., Satgé J., Sentenac-Roumanou H., Fatôme M., Laval J.D., Lion C., Alazard O., Chabertier P. (1994) Synthesis and radiopharmacological study of new metalated thiazolidines and dithioacetals of $\mathrm{N}$-allyl-substituted cysteamine and methylcysteamine, Appl. Organomet. Chem. 8, 481-490.

Rima G., Satgé J., Sentenac-Roumanou H., Fatôme M., Laval J.D., Lion C., Dagiral R. (1996) New germaheterocycles, germadithioacetals and a germylated oxide and sulfide derived from cysteamine, methylcysteamine and $N$-substituted cysteamine: synthesis and radioprotective activity, Appl. Organomet. Chem. 10, 113-123.

Rima G., Satgé J., Sentenac-Roumanou H., Fatôme M., Laval J.D., Lion C., Thiriot C., Dagiral R., Martin C. (1997) Synthesis and radioprotective properties of new metallathiazolidines, dialkyloxathiagermolanes, dialkylgermocanes and metalladithioacetals, Main Group Met. Chem. 20, 255-267.

Rima G., Satgé J., Dagiral R., Lion C., Fatôme M., Roman V., Laval J.D. (1998) Synthesis and radioprotective activity of new organosilicon and germanium compounds, Metal-Based Drugs 5, 139-146.

Rima G., Satgé J., Dagiral R., Lion C., Fatôme M., Roman V., Laval J.D. (1999a) Synthesis and application of new organometallic compounds of silicon and germanium in chemical radioprotection, Appl. Organomet. Chem. 13, 583-594.

Rima G., Satgé J., Dagiral R., Lion C., Sentenac-Roumanou H., Fatôme M., Roman V., Laval J.D. (1999b) A review on the radioprotective activity of organogermanium and organosilicon compounds, Metal-Based Drugs 6, 49-60.

Satgé J., Cazes A., Bouchaut M., Fatôme M., Sentenac-Roumanou H., Lion C. (1982) Synthesis and radioprotective activity of germa- and silathiazolidines, Eur. J. Med.Chem. 17, 433-436.

Satgé J., Rima G., Fatôme M., Sentenac-Roumanou H., Lion C. (1989) Syntheses and radioprotective activities of germatranes, silatranes, germylated oxides and sulfides with cysteamine, methylcysteamine and $\mathrm{N}$-(2-thioethyl)-1,3-diaminopropane hydrochloride ligands, Eur. J. Med. Chem. 24, 48-54.

Washburn L.C., Carlton J.E., Hayes R.L., Yuhas J.M. (1974) Distribution of WR 2721 in normal and malignant tissues of mice and rats bearing solid tumors. Dependence on tumor type, drug dose, and species, Radiat. Res. 59, 475-483.

Washburn L.C., Rafter J.J., Hayes R.L., Yuhas J.M. (1976) Prediction of the effective radioprotective dose of WR-2721 in humans through an interspecies tissue distribution study, Radiat. Res. 66, $100-105$.

Yuhas J.M. (1970) Biological factors affecting the radioprotective efficiency of S-2-[2aminopropylamino] ethylphosphorothioic acid (WR-2721). $\mathrm{LD}_{50} / 30 \mathrm{j}$ doses, Radiat. Res. 44, 621-628.

Yuhas J.M. (1972) Improvement of lung tumor radiotherapy through differential chemoprotection of normal and tumor tissue, J. Natl-Cancer Inst. 48, 1255-1257.

Yuhas J.M. (1973) Radiotherapy of experimental lung tumors in the presence and absence of a radioprotective drug, S-2-(3-aminopropylamino)ethylphosphorothioic acid (WR-2721), J. NatlCancer Inst. 50, 69-78. 